\title{
Proximal Femoral Cortical Thickness and Medullary Canal Diameter in Soft and Hard Water Regions
}

\author{
Ahmed Elmorsy ${ }^{1 *}$, Sarah Whitehouse ${ }^{2}$, John Timperley ${ }^{3}$, Stephen Veitch ${ }^{4}$ \\ ${ }^{1}$ Royal Surrey County Hospital, Guildford, UK \\ ${ }^{2}$ Institute of Health and Biomedical Innovation, Queensland University of Technology, Brisbane, Australia \\ ${ }^{3}$ Royal Devon \& Exeter Hospital, Exeter, UK \\ ${ }^{4}$ Salisbury district Hospital, Salisbury, UK \\ Email:"ahmedelmorsy@doctors.org.uk,s.whitehouse@qut.edu.au, Alison.lake2@nhs.net, \\ stephen.veitch@salisbury.nhs.uk
}

Received 30 January 2016; accepted 19 March 2016; published 22 March 2016

Copyright (C) 2016 by authors and Scientific Research Publishing Inc.

This work is licensed under the Creative Commons Attribution International License (CC BY).

http://creativecommons.org/licenses/by/4.0/

(c) (i) Open Access

\begin{abstract}
This study compared proximal femoral morphology in patients living in soft and hard water regions. The proximal femoral morphology of two groups of 70 patients living in hard and soft water regions with a mean age of 72.29 (range: 50 to 87 years) was measured using an antero-posterior radiograph of the non-operated hip with magnification adjusted. The medullary canal diameter at the level of the lesser trochanter (LT) was significantly wider in patients living in the hard water region (mean width: $1.9 \mathrm{~mm}$ wider; $p=0.003$ ). No statistical significant difference was found in the medullary canal width at $10 \mathrm{~cm}$ below the level of LT, Dorr index, or Canal Bone Ratio (CBR). In conclusion, the proximal femoral morphology does differ in patients living in soft and hard water areas. These results may have an important clinical bearing in patients undergoing total hip replacement surgery. Further research is needed to determine whether implant survivorship is affected in patients living in hard and soft water regions.
\end{abstract}

\section{Keywords}

Hip, Hard Water, Soft Water, Femoral Cortical Thickening, Medullary Diameter

\section{Introduction}

The senior author noticed that the femoral canals in patients living in a hard water region tended to be narrower ${ }^{*}$ Corresponding author. 
when preparing the femoral canal for total hip replacement (THR) compared to those living in soft water regions. In hard water regions, the calcium carbonate level is $>100 \mathrm{mg} / \mathrm{l} \&$ calcium level $>40 \mathrm{mg} / \mathrm{l}$ and in soft water regions, the calcium carbonate level is $<100 \mathrm{mg} / \mathrm{l} \&$ calcium level $<40 \mathrm{mg} / \mathrm{l}$ [1]. Communities living in a region of hard water are therefore receiving a supplement of dietary calcium. The serum calcium \& magnesium level is higher in those living in areas supplied by hard water [2]. High serum levels of calcium inhibit parathyroid hormone (PTH) release, which inhibits bone resorption [3]. Higher serum calcium levels also promote osteoblastic bone mineralization [4]. These effects lead to an increase in bone mass. Age, sex, and racial factors have been demonstrated to influence the femoral morphology [5]-[7], but no studies have looked at the influence of the hardness of water on the proximal femoral morphology. The aim of this study was to assess whether there was a difference in proximal femoral morphology in patients living in hard and soft water areas.

\section{Patients and Method}

A group of 114 Patients had undergone a primary Total Hip Replacement (THR) during the period from February 2010 to December 2012 under the care of the senior author at Salisbury District Hospital (SDH). All patients lived in a hard water region (Wiltshire, Hampshire and Dorset) and were selected for the hard water group (HARD Group). A group of 150 patients who had undergone a primary THR during the period from October 2009 to October 2010 at Royal Devon and Exeter Hospital (RDEH) living in a soft water region (Devon) (SOFT Group). As the far East Devon does contain moderately hard water patients living in this area were excluded. Patients were also excluded from both groups if they had bilateral THR, THR performed for a pathology other than primary osteoarthritis and all patients with inadequate $x$-ray films. Following the exclusion criteria; out of 114 patients in the HARD group, 26 patients excluded due to in adequate post X-ray films, 16 excluded due to having bilateral THR, and 2 patients excluded because they had their THR due to other causes than primary osteoarthritis. In the SOFT Group; out of 150 patients, 37 patents excluded due to in adequate post operative x ray films, 40 patients excluded due to having bilateral THR, one patient excluded due to having THR for other cause than primary Osteoarthritis and 2 patients excluded because the first post operative $\mathrm{x}$ ray was done more than 12 months after the operation's date. So, there were 70 patients (51 females, 19 males) mean age 72.24 (range: 50 84) in the HARD group and 70 patients (45 females, 25 males) mean age 72.34 (range: 52 to 87) in the SOFT group.

The hardness of water supplied to each group was confirmed by the South West Water company [1]. An AP digital radiograph of both hips taken within 12 months following THR was corrected to the actual size of the femoral head prosthesis by adjusting the magnification using PACS (Centricity Enterprise Web V 3.0). All measurements were performed on the non operated side. The mean time between the date of the operation and the date when the patient had the examined X-ray was 42 days in the HARD Group, with 53 patients (75.7\%) having their X-rays within a week postoperatively. In the SOFT group, the mean time was 18 days with 65 patients (92.8\%) having their examined post operative X-ray within a week of surgery.

A reference line was made through the mid-point of the lesser trochanter (LT) and a line perpendicular to the long axis of the femur was drawn. Further lines were drawn perpendicular to the anatomical axis of the femur at $7 \mathrm{~cm}$ and $10 \mathrm{~cm}$ below the LT. Measurements of the medullary canal width were made at the level of the mid-point of the lesser trochanter, $10 \mathrm{~cm}$ distal to the centre of LT, and the external diameter of the cortex at 10 $\mathrm{cm}$ below LT. Using these measurements the Dorr index was calculated from the ratio between the medullary canal diameter at the level of LT and at 10 below it [8] and the Canal bone ratio (CBR) which is the ratio of the endosteal diameter to the external diameter of the femur at $10 \mathrm{~cm}$ below the centre of LT [9] (Figure 1).

Following confirmation of normality, data were analysed using t-tests and presented as means and $95 \%$ confidence intervals. Frequencies were compared using the chi-squared test. The significance level was set at 5\%, which meant that following adjustment for multiple testing (6 variables) using Bonferroni's correction, the p-value to indicate statistical significance at the $5 \%$ level was $0.0083(0.05 / 6)$.

\section{Results and Discussion}

The HARD group consisted of 51 females and 19 males with a mean age of 72.24 years (range: 50 - 84). There were $42(60 \%)$ performed on the right side. The SOFT group consisted of 45 females and 25 males with a mean age 72.34 years (range: 52 to 87 ), and 38 (54.3\%) performed on the right. There was no significant difference between the groups for age $(\mathrm{p}=0.327)$ or gender $(\mathrm{p}=0.276)$. All patients had undergone a unilateral cemented 


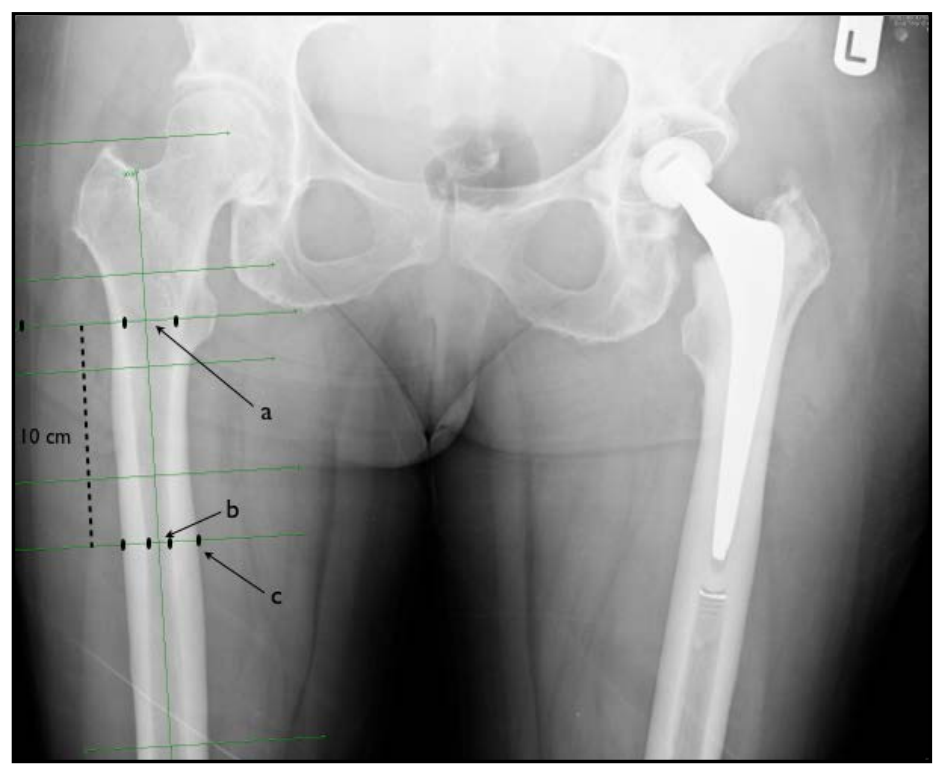

Figure 1. Plain radiograph of both hips, A-P view. a: Medullary canal width at he level of the geometric centre of LT. b: Medullary canal width at the level of $10 \mathrm{~cm}$ distal to the centre of LT. c: External cortical diameter at the level of $10 \mathrm{~cm}$ distal to the centre of LT.

Total Hip Replacement using the cemented Exeter Hip Replacement (Stryker Orthopedics, Mahwah, NJ). The size of the femoral head used to adjust for magnification differed between groups $(p<0.001)$. In the SOFT group, 50 patients had size $28 \mathrm{~mm}$ heads, 18 had size $32 \mathrm{~mm}$ heads and 2 had size $36 \mathrm{~mm}$ heads. In the HARD group, 4 patients had size $28 \mathrm{~mm}$ heads and 66 had size $32 \mathrm{~mm}$ heads. The medullary canal width at the level of LT in the HARD group had a mean of $26.0 \mathrm{~mm}$ (range: 25.1 to 27.0) and the SOFT group had a mean of 24.1 $\mathrm{mm}$ (range: 23.2 to 25.0). The mean difference between the groups was $1.9 \mathrm{~mm}$ and this was statistically significant $(\mathrm{p}=0.003)$. There was no difference in the medullary canal diameters $10 \mathrm{~cm}$ below the centre of the LT (p $=0.194)$, the Dorr index $(\mathrm{p}=0.135)$, or the CBR $(\mathrm{p}=0.099)$ (see Table 1$)$. In hard water regions, the calcium carbonate level is $>100 \mathrm{mg} / \mathrm{l} \&$ calcium level $>40 \mathrm{mg} / \mathrm{l}$ and in soft water regions, the calcium carbonate level is $<100 \mathrm{mg} / \mathrm{l} \&$ calcium level $<40 \mathrm{mg} / \mathrm{l}$ [1]. Communities living in a region of hard water are therefore receiving a supplement of dietary calcium. The most metabolically active bone in the proximal femur is in the region of the femoral neck and adjacent to the LT where there is a higher proportion of cancellous bone compared to the proximal femur $10 \mathrm{~cm}$ below the LT. The findings of our study support the effect that a high calcium intake has on bone metabolism. Those patients living in a hard water region have an increased mean width of $1.9 \mathrm{~mm}$ at the level of the lesser trochanter which is a region containing a high proportion of metabolically active cancellous bone. No difference was found in the width at $10 \mathrm{~cm}$ distal to the LT where there is a lower proportion of cancellous bone, Dorr index, or in the proportion of cortical to cancellous bone measured using the Canal Bone Ratio (CBR) at $10 \mathrm{~cm}$ distal to the LT [10].

Medullary canal width at the level of LT and $10 \mathrm{~cm}$ below LT were chosen as they are the most popular and validated areas to measure in the proximal femur and are used to calculate the Dorr Index and Canal Bone Ratio. The Dorr index looks at the overall morphology of the femur [8]. The Canal Bone Ratio (CBR) reflects the cortical bone thickness at $10 \mathrm{~cm}$ below LT and has been shown to have a direct relationship to the bone mineral density t-score in the proximal femur [9]. When the endosteal diameter is 0.49 or more compared to the external diameter, it is likely that the proximal femur is osteoporotic [9]. In this study CBR ranges from 0.3 to 0.62 in HARD group with 8 patients scoring CBR more than 0.49. In SOFT group CBR ranges from 0.59 - 0.58, with only 8 patients scoring CBR more than 0.49 .

The groups studied were all those who had undergone a total hip replacement within a specified period. The replaced femoral head of a known size was used as the measuring template on radiographs in order to accurately measure the proximal femur of the non-operated hip. The femoral head size differed between the SOFT and HARD groups with the SOFT group predominantly having a $28 \mathrm{~mm}$ head and the HARD group a $32 \mathrm{~mm}$ head. 
Table 1. Mean (95\% confidence intervals) measurements of the proximal femoral morphology.

\begin{tabular}{cccc}
\hline Variable & Hard group & Soft group & p-value \\
\hline $\begin{array}{c}\text { Medullary canal (M/C) width at Lesser } \\
\text { trochanter (LT) in mm) }\end{array}$ & 26.0 & 24.1 & 0.003 \\
M/C width at 10 cm below LT in mm & $(25.09$ to 26.93$)$ & $(23.16$ to 24.97$)$ & \\
& 11.9 & 11.5 & \\
External diameter at 10 below LT in mm & $(11.43$ to 12.34$)$ & $(10.99$ to 11.93$)$ & 0.194 \\
& 27.8 & 27.9 & 0.718 \\
DORR INDEX & 0.46 & $(27.18$ to 28.68$)$ & 0.135 \\
Canal bone ratio & $(0.44$ to 0.48$)$ & $(0.46$ to 0.51$)$ & 0.099 \\
\hline
\end{tabular}

The difference in choice of head size was due to surgeon preference. The radiographic magnification was individually adjusted to the known head size in order to avoid measurement bias.

One limitation of this study was that we did not know how long patients had lived in areas of hard and soft water and there is no available information regarding their diet and life style. Another limitation is that a number of patients were excluded due to inadequate radiographs of the femur, which prevented measurements at $10 \mathrm{~cm}$ below the lesser trochanter. The choice of femoral measurements was made based on those that were validated and regularly used in the literature. There are now technologies available whereby the whole proximal femur can be digitally scanned and measured to allow a more detailed and accurate comparison between groups of patients. In view of the findings of this study future research using these technologies is important to more precisely quantify the differing morphology in patient living in hard and soft water areas.

These results may have an important clinical bearing in patients undergoing total hip replacement surgery. The stability of the femoral component in uncemented implants depends on the balance of proximal and distal load transfer from the implant to the femur [11]. The relative contributions of the proximal and distal support depend mainly on the fit of the prosthesis to the bone. As patients in HARD water areas have a wider metaphysis, this may affect the initial stability required for certain types of uncemented implants and lead to earlier failure. Further research is also needed in patients who have undergone joint replacement to determine whether implant survivorship is affected in patients living in hard and soft water regions.

\section{References}

[1] South West Water. http://www.southwestwater.co.uk/index.cfm?articleid=11233\&searchkey=hard\%20water

[2] Bierenbaum, M.L., Fleischman, A.I., Dunn, J.P., et al. (1972) Serum Lipids in Hard and Soft Water Communities. Israel Journal of Medical Science, 5, 567.

[3] Karp, H.J., Ketola, M.E. and Lamberg-Allardt, C.L.E. (2009) Acute Effect of Calcium Carbonate, Calcium Citrate and Potassium Citrate on Markers of Calcium and Bone Metabolism in Young Women. British Journal of Nutrition, 102, 1341-437. http://dx.doi.org/10.1017/S0007114509990195

[4] Bonjour, J.P. (2011) Calcium and Phosphate: A Duet of Ions Playing for Bone Health. Journal of the American College of Nutrition, 30, 438S-448S. http://dx.doi.org/10.1080/07315724.2011.10719988

[5] Smith Jr., R.W. and Walker, R.R. (1964) Femoral Expansion in Raging Women: Implications for Osteoporosis and Fractures. Science, 145, 156-157. http://dx.doi.org/10.1126/science.145.3628.156

[6] Ruff, C.B. and Hayes, W.C. (1984) Age Changes in Geometry and Mineral Content of the Lower Limb Bones. Annals of Biomedical Engineering, 12, 573-584. http://dx.doi.org/10.1007/BF02371450

[7] Ericksen, M.F. (1979) Aging Changes in the Medullary Cavity of the Proximal Femur in American Blacks and Whites. American Journal of Physical Anthropology, 51, 563-569. http://dx.doi.org/10.1002/ajpa.1330510408

[8] Dorr, L.D. (1993) Structural and Cellular Assessment of Bone Quality of Proximal Femur. Bone, 14, $231-242$. http://dx.doi.org/10.1016/8756-3282(93)90146-2

[9] Yeung, Y., Chiu, K.Y., Yau, W.P., Tang, W.M., Cheung, W.Y. and Ng, T.P. (2006) Assessment of the Proximal Femoral Morphology Using Plain Radiograph—Can It Predict the Bone Quality? The Journal of Arthroplasty, 21, 408413. 
[10] Noble, P.C., Box, G.G., Kamaric, E., Fink, M.J., Alexander, J.W. and Tullos, H.S. (1998) The Effect of Aging on the Shape of the Proximal Femur. Clinical Orthopaedics and Related Research, 319, 31-44.

[11] Huiskes, R. (1980) Stress Analyses of Implanted Orthopaedic Joint Prostheses for Optimal Design and Fixation. Acta Orthopaedica Belgica, 46, 711-727. 